\title{
Heavy Flavour spectroscopy and exotica with CMS
}

\author{
Alexis Pompili* \\ Università degli Studi Aldo Moro di Bari and I.N.F.N. Sezione di Bari \\ E-mail: alexis.pompili@ba.infn.it
}

In the last 13 years the discovered quarkonium-like states have renewed the interest in hadron spectroscopy and the LHC experiments are highly contributing to this field. Two relevant contributions by the CMS Collaboration to the exotic heavy flavour spectroscopy are discussed. The first study concerns the production of the $X(3872)$, either prompt or from beauty hadron decays. The cross-section ratio of the $X(3872)$ with respect to the $\psi(2 S)$ in the $J / \psi \pi^{+} \pi^{-}$decay channel and the fraction of $X(3872)$ coming from $B$-hadron decays are measured as a function of transverse momentum $\left(p_{\mathrm{T}}\right)$, covering unprecedentedly high values of $p_{\mathrm{T}}$. Moreover the prompt $X(3872)$ cross section times branching fraction is extracted differentially in $p_{\mathrm{T}}$, for the first time in central rapidity region, and compared to the theoretical predictions available. Finally the dipion invariant mass spectrum of the $J / \psi \pi^{+} \pi^{-}$system, in the $X(3872)$ decay, is also investigated. The second study concerns the study of the $B^{ \pm} \rightarrow J / \psi \phi K^{ \pm}$decays. A peaking structure in the $J / \psi \phi$ invariant mass spectrum is observed near the kinematic threshold and evidence for an additional peaking structure at higher $J / \psi \phi$ mass is also reported. Fitting the first structure by means of an S-wave relativistic Breit-Wigner line shape above a three-body phase-space component, representing the residual non-resonant background after background subtraction, provides a signal statistical significance exceeding five standard deviations. The fitted mass and width values are $m=4148.0 \pm 2.4$ (stat.) \pm 6.3 (syst.) $\mathrm{MeV}$ and $\Gamma=28_{-11}^{+15}$ (stat.) \pm 19 (syst.) MeV, respectively. This study is a confirmation of the $Y(4140)$ state discovered by the CDF Collaboration. A preliminary study of possible reflections indicates that this peaking structure, unlike the additional one, may be not correlated with an excess, with respect to the pure phase-space profile, appreciated in the region of the $\phi K^{ \pm}$mass spectrum where a couple of interfering large resonances might appear. However understanding the nature of these peaking structures would require a proper full amplitude analysis that can be performed only extending the study to the full available data sample.

Fourth Annual Large Hadron Collider Physics

13-18 June 2016

Lund, Sweden

* Speaker.

On behalf of the CMS Collaboration. 


\section{INTRODUCTION}

In the seventies of last century, with the development of QCD theory, it became clear that there was no fundamental reason why only three-quarks and quark-antiquark combinations should exist [1] excluding other colorless bound quarks states or even glueballs (bound gluons states) and hybrids (bound states of gluons and quarks). Exotic hadrons would be particles made by quarks (and eventually gluons) not having the same quark content as ordinary hadrons and thus not fitting into their usual scheme. Exotic multi-quark states have been long predicted in the light scalar sector where however it has been difficult to differentiate them from conventional states. Instead the charmonium system, having well-predicted spectrum and distinct properties (zero-charge, zerostrangeness, constrained decay transitions), is suitable to look for exotic states that can be charged $(c \bar{c} u \bar{d})$, strange $(c \bar{c} d \bar{s})$ or both $(c \bar{c} u \bar{s})$.

The charmonium mesons spectrum is well described by QCD quark-potential models. Opencharm thresholds $\left(D \bar{D}, D \bar{D}^{*}, D^{*} \bar{D}^{*}, \ldots\right)$ play an important role: below them, the states should be narrow resonances slowly decaying into non-charmed mesons or dilepton pairs, whereas, above them, the states should be large resonances rapidly decaying mainly into charmed mesons pairs. Charmonium spectrum and properties are well understood up to the $\psi(3770)$; the states experimentally found can be perfectly identified with the levels predicted by quark-potential models. For instance the difference between the spin-averaged centroid mass of the $1 P$ triplet and the $h_{c}(1 P)$ mass is about $1 \mathrm{MeV}$ namely in agreement with hyperfine mass splitting calculation.

In the last 13 years, starting from the $X$ (3872) observation [2], many unexpected states were observed, either at $B$-factories and/or at hadron colliders, while decaying to conventional charmonium in spite of being above the open-charm thresholds. These charmonium-like states are inconsistent with the expected $(c \bar{c})$ spectrum. Indeed mass values do not fit the calculated levels (however thresholds might deform the spectrum), widhts are surprisingly narrow and many experimental decay rates do not agree with those expected. Few of these states were subsequently adopted into the existing $(c \bar{c})$ scheme, some others remained in a limbo (not confirmed by a second experiment), and many of them, even if established, still remain a puzzle (sometimes quantum numbers are not experimentally determined yet) [3]. Analogue states in the bottomonium sector have been found as well.

A theoretical framework describing all or the majority of these so-called XYZ mesons is missing. To explain their nature several alternative models (such as tetraquarks and hadron molecules) were introduced and they can be classified according to their constituents and how they are clustered within the meson. Essentially tetraquarks are compact bound states made of a diquark-antidiquark pair whereas molecules are weakly bound states formed by 2 hadrons.

There are two main production processes of the charmonium-like states at hadron machines: a) prompt production $p p(p \bar{p}) \rightarrow(c \bar{c})^{*}+$ other, b) nonprompt production in b-jets. In the former case an inclusive search is needed whereas in the latter they are tipically searched for in exclusive beauty meson decays $\left(B \rightarrow(c \bar{c})^{*}+\right.$ other $)$. Establishing their existence with both production mechanisms would be ideal but inclusive searches are experimentally difficult because of the high backgrounds and the low trigger bandwidth for prompt muons at low transverse momenta.

Typical decay processes in which they can be searched for are mainly: a) hadronic transition to a lighter $c \bar{c}$ meson through the emission of light hadrons $(\pi, \pi \pi, \rho, \phi)$, b) electromagnetic tran- 
sition to a lighter $c \bar{c}$ meson through the emission of photon. The former are suitable for triggering on dimuons $(J / \psi, \psi(2 S))$ but still difficult to reconstruct with low backgrounds without having an hadronic particle identification (as in CMS). The latter are challenging because of the need of pair converted low energy photons that are characterized by a very low reconstruction efficiency.

The analyses of LHC Run-I data are contributing to provide new experimental observations and measurements of exotic mesons. CMS has contributed to this new spectroscopy by providing some important results. Among them it is worthy to mention the study of the production of $X(3872)$ [4], the confirmation [5] of the $Y(4140)$ state first seen by CDF and the search for a neutral bottomonium partner of the $X$ (3872) [6]. Right after this LHCP conference CMS has released [7] a preliminary upper limit on the relative (with respect to $B_{s}^{0}$ ) production rate of $X(5568)$, the state recently claimed by D0 [8], multiplied by the unknown branching fraction of the $X(5568)^{ \pm} \rightarrow B_{s}^{0} \pi^{ \pm}$ decay. The first two contributions among those just mentioned will be discussed in the two subsequent sections. The study of the inclusive $X(3872)$ production in $p p$ collisions, at $\sqrt{s}=7 \mathrm{TeV}$, is presented in Section 2, whereas the observation of two peaking structures in the $J / \psi \phi$ invariant mass spectrum from $B^{ \pm} \rightarrow J / \psi \phi K^{ \pm}$decays is discussed in Section 3 .

\section{Study of the inclusive $X(3872)$ production in $p p$ collisions at $\sqrt{s}=7 \mathrm{TeV}$}

$X(3872)$ is the first exotic state discovered by Belle [2] in the $B^{+} \rightarrow X(3872) K^{+} \rightarrow J / \psi \pi^{+} \pi^{-} K^{+}$ decays and confirmed by CDF [9] with an inclusive search in $p \bar{p}$ collisions (production was found to be mainly prompt since only $\simeq 16 \%$ of $X(3872)$ are from beauty-hadron decays). As soon as LHC started this state was confirmed by LHCb [10], CMS [4] and very recently by ATLAS [11].

The CMS study is performed on the data recorded in 2011, corresponding to an integrated luminosity of $4.8 \mathrm{fb}^{-1}$, looking inclusively for the $X(3872)$ through its decays into $J / \psi \pi^{+} \pi^{-}$, with the subsequent decay of the $J / \psi$ into a pair of muons. The experimental signature of this decay channel in CMS [12] is clean enough thanks to the high granularity of the tracker and the high efficiency of $J / \psi$ identification. The data are collected with requirements on the dimuon system imposed at the trigger level, such as rapidity within 1.25 and a transverse momentum threshold of $9.9 \mathrm{GeV}$ in order to benefit from a better mass resolution in the central rapidity region. The analysis is thus performed in the $p_{\mathrm{T}}$ kinematic range of the $J / \psi \pi^{+} \pi^{-}$system between 10 and $50 \mathrm{GeV}$ and in the rapidity region $|y|<1.2$, collecting about $12000 X(3872)$ candidates. Details about the event selection and the event simulations used to determine acceptances and efficiencies are described in Ref.[4]. The $X(3872)$ was assumed to be an unpolarized state and its $J^{P C}$ fixed at $1^{++}$value, as favoured by the existing LHCb study [13] and later confirmed by the same experiment with a full angular analysis unambiguously determining its quantum numbers [14].

\subsection{Measurement of the $X(3872)$ cross section ratio with respect to the $\psi(2 S)$}

The ratio of the $X(3872)$ and $\psi(2 S)$ cross sections times their branching fractions to $J / \psi \pi^{+} \pi^{-}$ is obtained from the measured numbers of signal events, $N_{X(3872)}$ and $N_{\psi(2 S)}$, and correcting for the efficiencies $(\varepsilon)$ and acceptances $(A)$, according to

$$
R=\frac{\sigma(p p \rightarrow X(3872)+\text { anything }) \cdot B\left(X(3872) \rightarrow J / \psi \pi^{+} \pi^{-}\right)}{\sigma(p p \rightarrow \psi(2 S)+\text { anything }) \cdot B\left(\psi(2 S) \rightarrow J / \psi \pi^{+} \pi^{-}\right)}=\frac{N_{X(3872)} \cdot A_{\psi(2 S)} \cdot \varepsilon_{\psi(2 S)}}{N_{\psi(2 S)} \cdot A_{X(3872)} \cdot \varepsilon_{X(3872)}}
$$



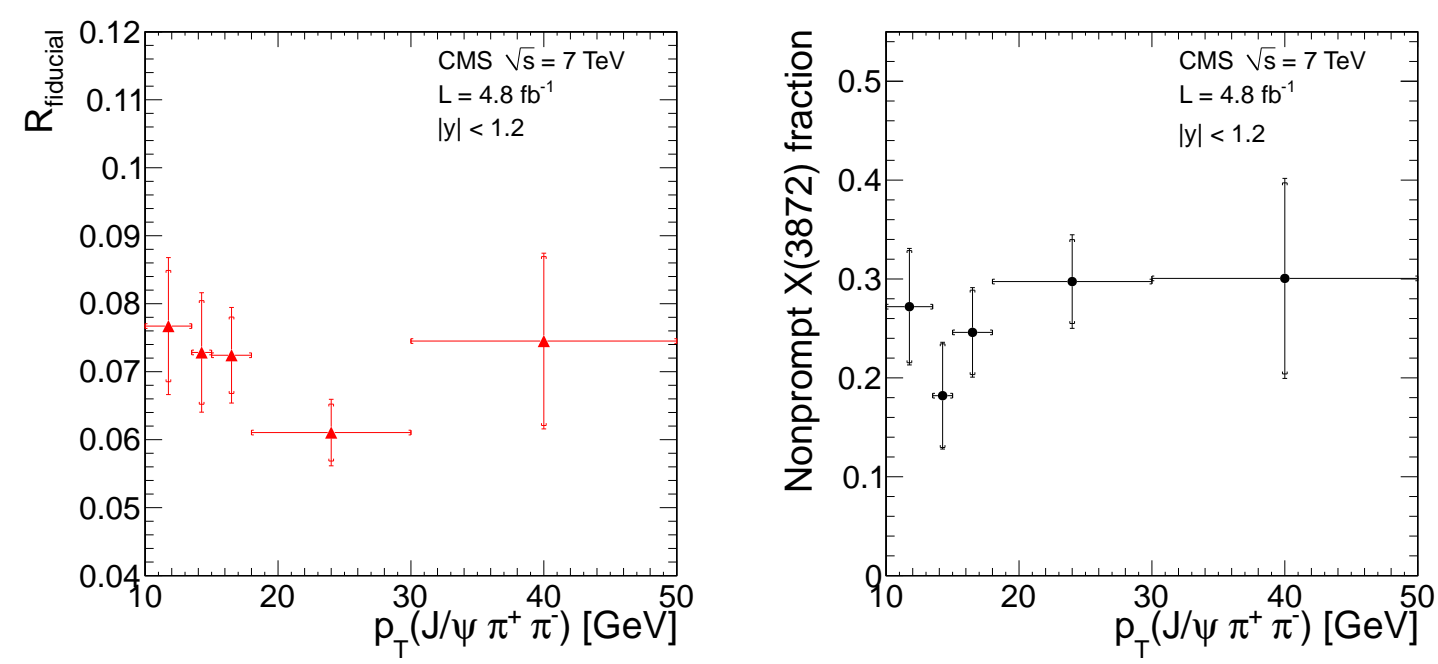

Figure 1: Left: ratio between $X(3872)$ and $\psi(2 S)$ cross sections times branching fraction as a function of $p_{\mathrm{T}}$. Outer error bars are the total uncertainty while inner error bars indicate statistical uncertainty only. The data points are placed at the centre of each $p_{\mathrm{T}}$ bin. Right: measured $X(3872)$ nonprompt fraction, uncorrected for acceptance, as a function of $p_{\mathrm{T}}$; error bars and data point placing are as in the left plot.

The signal yields, $N_{X(3872)}$ and $N_{\psi(2 S)}$, are determined from unbinned maximum likelihood fits to the invariant mass spectra of the $J / \psi \pi^{+} \pi^{-}$system, separately for the $X(3872)$ and $\psi(2 S)$ in different mass windows. The acceptances and efficiencies of the $X(3872)$ and $\psi(2 S)$ final states are determined from the simulation; acceptance is estimated assuming both $X(3872)$ and $\psi(2 S)$ states unpolarized (and no systematic uncertainty is assigned to cover other cases). Monte Carlo simulation studies are performed to determine the systematic uncertainties and a data-driven method is used to verify the efficiencies, as described in Ref.[4]. Many systematic uncertainties cancel out in the ratio; the main one arises from the limited knowledge of the $X(3872)$ transverse momentum. The total uncertainty for each $p_{\mathrm{T}}$ interval is typically $10 \%$ statistical and $5-13 \%$ systematic; no significant dependence on $p_{\mathrm{T}}$ is observed.

Given the unknown production mechanism of the $X(3872)$, a result without acceptance corrections for the muon and pion pairs has been provided in a fiducial region (phase-space window defined by kinematical cuts on the muons and pions). Left plot in Figure 1 shows the ratio between the $X(3872)$ and $\psi(2 S)$ cross sections times branching fractions, $R_{\text {fiducial }}=N_{X} / N_{\psi(2 S)} \cdot \varepsilon_{\psi(2 S)} / \varepsilon_{X}$, as a function of $p_{\mathrm{T}}$ : again no significant dependence on the $p_{\mathrm{T}}$ can be appreciated. Integrating over the interval $10<p_{\mathrm{T}}<50 \mathrm{GeV}$ an $R_{\text {fiducial }}=0.0694 \pm 0.0029$ (stat $) \pm 0.0036$ (syst $)$ is obtained.

\subsection{Measurement of the nonprompt fraction of $X(3872)$ production}

The fraction of $X(3872)$ produced from decays of beauty hadrons is referred to as nonprompt fraction. The prompt and nonprompt components of the $X(3872)$ candidates can be separated on the basis of the pseudo-proper decay length $\left(l_{x y}\right)[4]$ that is related to the distance between the primary vertex and the secondary one. The latter is formed by the tracks of the $J / \psi \pi^{+} \pi^{-}$ system whereas the former is selected, among those associated to the six (in average) $p p$ collisions 
occuring in the same bunch crossing, as the one that minimizes the distance from the secondary vertex along the beam direction. A $B$-hadron-enriched sample is obtained requiring $l_{x y}$ larger than $100 \mu \mathrm{m}$, with an estimated (from simulation) contribution by prompt $X(3872)$ candidates below $0.1 \%$.

The nonprompt fraction is then obtained from the ratio between the signal yield extracted from this $B$-hadron-enriched sample and the signal yield in the inclusive sample, after correction for the efficiencies of the decay length selection criteria determined from simulations. Detailed studies are performed to verify the stability of the results and to determine the systematic uncertainties leading to a total systematic uncertainty of $6-10 \%$; thus the total uncertainty of the measurement is dominated by its statistical component $(\sim 20 \%)$. The $X(3872)$ nonprompt fraction is estimated to be $\simeq 0.263 \pm 0.028$ which implies that the total cross section is quite dominated by prompt production. This integrated value is significantly smaller than that for the $\psi(2 S)$ [15]. As shown in Figure 1 (right), the $X(3872)$ nonprompt fraction has no significant dependence on $p_{\mathrm{T}}$ while that for $\psi(2 S)$ increases with it [15].

\subsection{The differential and total prompt cross section of $X(3872)$ production}

By exploiting the measurements described above the prompt production cross section for the $X(3872)$ times the unknown branching fraction for the decay of $X(3872)$ to $J / \psi \pi^{+} \pi^{-}$has been determined through the expression

$\sigma_{X}^{P R} \cdot B\left(X \rightarrow J / \psi \pi^{+} \pi^{-}\right)=\frac{1-f_{X}^{B}}{1-f_{\psi(2 S)}^{B}} \cdot R \cdot\left[\sigma_{\psi(2 S)}^{P R} \cdot\left(B\left(\psi(2 S) \rightarrow \mu^{+} \mu^{-}\right)\right] \cdot \frac{B\left(\psi(2 S) \rightarrow J / \psi \pi^{+} \pi^{-}\right)}{B\left(\psi(2 S) \rightarrow \mu^{+} \mu^{-}\right)}\right.$

where $R$ is the cross section ratio discussed in Section 2.1 and $f_{X}^{B}$ the nonprompt fraction of Section 2.2 , whereas prompt $\psi(2 S)$ cross section and the nonprompt $\psi(2 S)$ fraction are borrowed from a previous CMS measurement [15]. The latter measurement was performed using the $\psi(2 S) \rightarrow$ $\mu^{+} \mu^{-}$decay mode and provides results as a function of transverse momentum up to $30 \mathrm{GeV}$, in the same rapidity range of this analysis. Finally, in this calculation, the branching fraction of the $\psi(2 S) \rightarrow J / \psi \pi^{+} \pi^{-}$is taken from the PDG [16], whereas that for its decay in two muons is taken to be equal to the one in two electrons, which is more precisely known, again taken from Ref.[16].

By means of this combination, the differential cross section for prompt $X(3872)$ production times the branching fraction is obtained as a function of $p_{\mathrm{T}}$, as shown in Figure 2 (left), in the central rapidity region $|y|<1.2$. The main sources of systematic uncertainty are related to the measurement of $R$ and of the prompt $\psi(2 S)$ cross section. This result is compared to a theoretical prediction based on NRQCD factorization approach [17] with calculations normalized using Tevatron results and modified by the authors to match the CMS phase-space. While the shape is reasonably well described by the theory, the predicted cross section is much larger than observed in data. This theoretical prediction assumes, within an S-wave molecular model, the relative momentum of the mesons being bound by an upper limit of $400 \mathrm{MeV}$, which is quite high for a loosely bound molecule but it is considered possible as a result of significant rescattering effects. On the other hand an upper limit lower by one order of magnitude would imply prompt production rates lower by a few orders of magnitude [18].

The integrated prompt $X(3872)$ cross section times branching fraction for the kinematic region $10<p_{\mathrm{T}}<30 \mathrm{GeV}$ and $|y|<1.2$ is also determined to be $\sigma_{X}^{P R} \cdot B\left(X \rightarrow J / \psi \pi^{+} \pi^{-}\right)=$ 
$1.06 \pm 0.11$ (stat.) \pm 0.15 (syst.) nb, namely significantly below the theoretical prediction in the same kinematic region, which is $4.01 \pm 0.88 \mathrm{nb}$ [17].
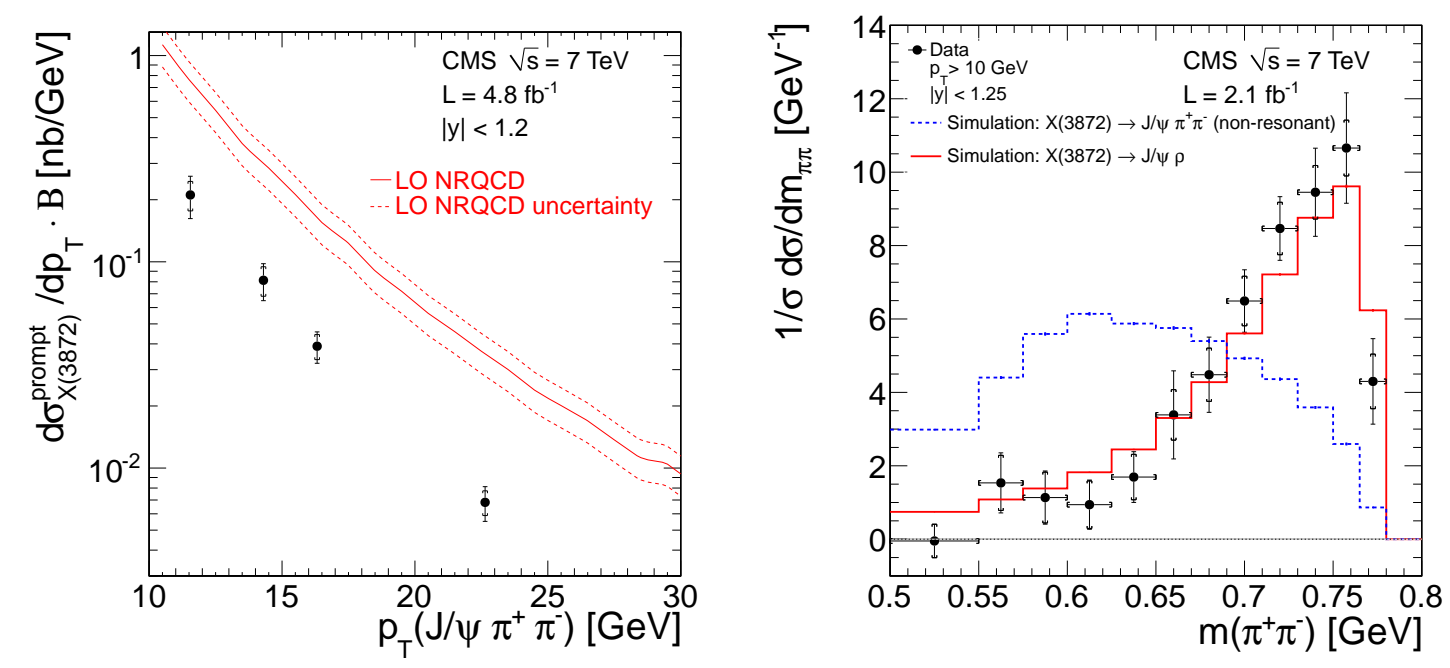

Figure 2: Left: measured differential cross section for prompt $X$ (3872) production times branching fraction of $X(3872) \rightarrow J / \psi \pi^{+} \pi^{-}(B)$ as a function of $p_{\mathrm{T}}$. The inner error bars indicate the statistical uncertainty while the outer error bars represent the total uncertainty. Theoretical prediction as explained in the text is shown by the solid line with the dotted line representing the uncertainty. The data points are placed where the theoretical value is equal to its mean value over each bin. $X(3872)$ and $\psi(2 S)$ are assumed unpolarized. Right: dipion invariant mass spectrum corrected for acceptance and efficiency and compared to prediction from simulation with (solid line) and without (dotted line) an intermediate $\rho^{0}$ decay. Again the inner(outer) error bars indicate the statistical(total) uncertainty.

\subsection{Study of the properties of the $X(3872)$ decay to $J / \psi \pi^{+} \pi^{-}$}

The dipion invariant-mass distribution from $X(3872)$ decays to $J / \psi \pi^{+} \pi^{-}$has been measured in order to investigate the decay properties of the $X(3872)$ and evaluate the presence of an intermediate $\rho^{0}$ state in the decay, as was suggested by previous studies [19][20] and also expected (a favoured decay to $J / \psi \rho$ implies even C-parity that was already suggested by the BaBar studies of the $X(3872)$ radiative decays [21]). The measurement is made within the kinematic intervals $10<$ $p_{\mathrm{T}}<50 \mathrm{GeV}$ and $|y|<1.25$. Details on the extraction of the dipion invariant-mass spectrum, and subsequent corrections for detector acceptance and efficiency estimated from the simulation, are given in Ref.[4]. The main systematic uncertainties are related to the signal extraction $(10-20 \%)$ and to corrections due to acceptance and efficiency $(4-6 \%)$. The data spectrum, when compared to $X(3872)$ signal simulations with and without an intermediate $\rho^{0}$ in the $X(3872)$ decay, shows much better agreement assuming the presence of the $\rho^{0}$.

\section{A peaking structure near threshold in the $J / \psi \phi$ mass observed in $B^{ \pm} \rightarrow J / \psi \phi K^{ \pm}$}

The observation of the $Y(3940)$ state [22] in the $J / \psi \omega$ mass spectrum close to the threshold motivated the search of other states in the $J / \psi \phi$ mass spectrum with similar characteristics. By 
studying the $B^{ \pm} \rightarrow J / \psi \phi K^{ \pm}$decays CDF observed [23][24] the $Y(4140)$ structure close to the kinematic threshold of the $J / \psi \phi$ invariant mass and found the evidence for a second structure called $Y(4274)$. The $Y(4140)$ could be explained as a $\operatorname{cs} \bar{c} \bar{s}$ tetraquark or a $D_{s}^{*} \bar{D}_{s}^{*}$ loosely bound molecule that would be the strange partner of the $Y(3940)$ state (interpreted as a $D^{*} \bar{D}^{*}$ molecule), or also as a threshold enhancement or weak transition with a $D_{s}^{ \pm} \bar{D}_{s}^{\mp}$ rescattering. LHCb did not confirm the $Y$ (4140) and provided an upper limit to its production [25].

By studying the $B^{ \pm} \rightarrow J / \psi \phi K^{ \pm}$decays produced in $p p$ collisions at $\sqrt{s}=7 \mathrm{TeV}$ CMS has searched for the two peaking structures in the $J / \psi \phi$ mass spectrum [5]; the data sample, selected at High Level Trigger (HLT) stage on the basis of the dimuon decay mode of the $J / \psi$, corresponds to an integrated luminosity of $5.2 \mathrm{fb}^{-1}$.

\section{1 $B$ decay reconstruction and mass difference extraction}

The $J / \psi$ candidates are reconstructed by pairs of oppositely charged muons, identified according to the selection implemented by the HLT dimuon trigger. Its configuration requires the dimuon $p_{\mathrm{T}}>6.9 \mathrm{GeV}$ and the dimuon transverse flight length significance greater than 3 ; for the latest running period considered it also requires $p_{\mathrm{T}}>4 \mathrm{GeV}$ for each muon. The $B^{ \pm} \rightarrow J / \psi \phi K^{ \pm}$candidates are reconstructed by combining three additional good quality charged tracks, having $p_{\mathrm{T}}>1 \mathrm{GeV}$, well pointing to the displaced $J / \psi$ vertex, with a total charge of \pm 1 and with kaon mass assigned to them. The five tracks, with the $\mu^{+} \mu^{-}$system kinematically constrained to the $J / \psi$ mass nominal value, are required to form a good 3D vertex with $\chi^{2}$ probability greater than $1 \%$. The $K^{+} K^{-}$pair combination with lower mass must have a reconstructed mass $1.008 \mathrm{GeV}<m\left(K^{+} K^{-}\right)<1.035$ $\mathrm{GeV}$ to be considered as a $\phi$ candidate. Sideband subtracted $\phi$ signal shows that any contribution from non- $\phi$ components is negligible. The selection criteria (see Ref.[5] for details), designed to maximize the $B$ signal yield ( $2480 \pm 160$ candidates), were determined before examining the mass difference $\Delta m \equiv m\left(\mu^{+} \mu^{-} K^{+} K^{-}\right)-m\left(\mu^{+} \mu^{-}\right)$, that is the observable used to search for possibile structures in the $J / \psi \phi$ mass and investigated up to $1.568 \mathrm{GeV}$ in order to reject the reflection from $B_{s}^{0} \rightarrow \psi(2 S) \phi \rightarrow\left(J / \psi \pi^{+} \pi^{-}\right) \phi$ decays (when one pion is not reconstructed and the other misidentified as kaon).

Specific simulated samples have been produced to provide, as a function of $J / \psi \phi$ mass, a) the $B$ mass resolution, b) the absolute $B$ reconstruction efficiency, c) the $J / \psi \phi$ mass resolution. The first is needed by the background subtraction method applied, the second by the relative efficiency correction and the third to configure the Gaussian resolution function in the final $\Delta m$ fit.

After background subtraction, obtained by a $20 \mathrm{MeV}$-sized bin-wise method, the $\Delta m$ spectrum distribution is shown in Figure 3 (left), without relative efficiency correction yet. This distribution is consistent with the one that can be extracted by applying the alternative technique of sPlot [26]. The efficiency correction to be used is consistent with a 2D relative efficiency correction alternatively extracted over the Dalitz plot reconstructed, from a pure phase-space generated signal Monte Carlo, applying the same seletion criteria used in the data. In both cases the difference due to the alternative method is treated as systematic uncertainty.

\section{2 $\Delta m$ spectrum fit results}

The binned 1D-fit to the $\Delta m$ distribution, with the fit model taking into account the relative efficiency correction, is shown in Figure 3 (left). The fit model includes the three-body phase-space 

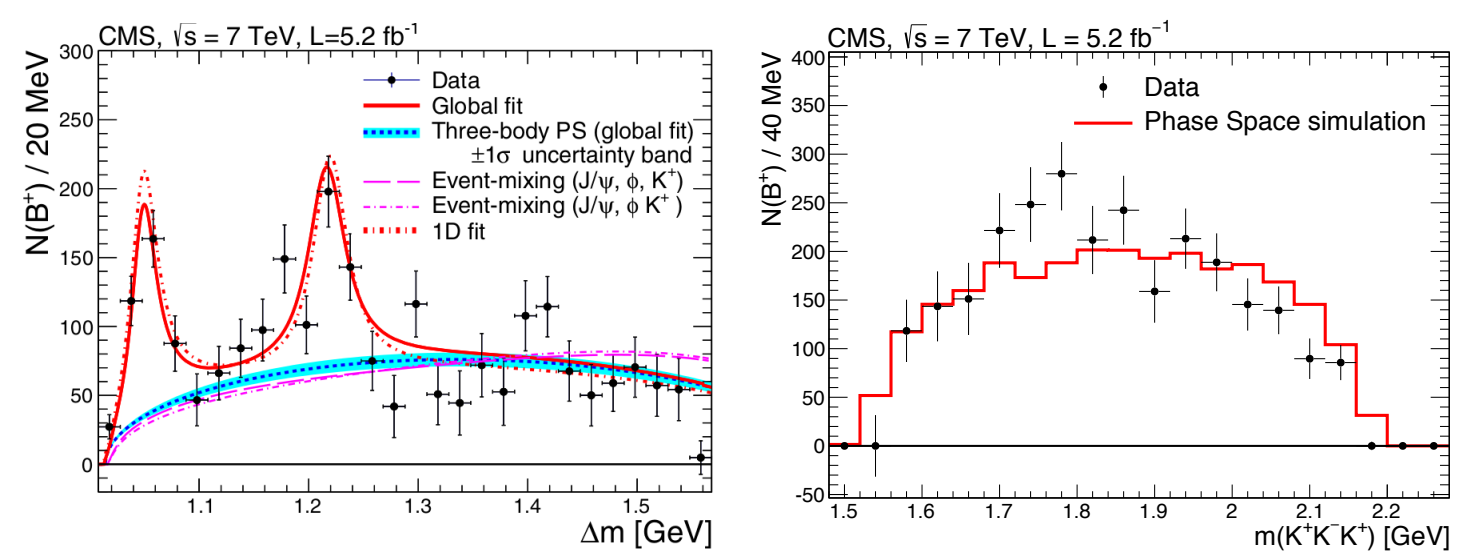

Figure 3: Left: background-subtracted $\Delta m$ distribution obtained as the number of $B^{ \pm} \rightarrow J / \psi \phi K^{ \pm}$candidates as a function of $\Delta m$. The superimposed 1D binned fit model is provided by the short-dashed red curve, whereas the solid curve is the global (2D) UML fit of the data. The dotted blue curve is the residual background contribution assumed to have a three-body phase space profile; the band represents the $\pm 1 \sigma$ uncertainty range for the background obtained from the global fit. The dashed and dash-dotted curves are background curves obtained from two different event-mixing procedures and normalized to the number of three-body phase space background events. Right: background-subtracted $\phi K^{ \pm}$invariant mass distribution; the solid red curve represents the predicted profile from the phase-space simulation.

profile for the residual incoherent background, whereas each structure is modelled with an S-wave relativistic Breit-Wigner function convolved with a Gaussian resolution function having width fixed to the value obtained from simulation. The mass resolution is about $1 \mathrm{MeV}$ at the threshold and smoothly increases with $\Delta m$ (it is about $4 \mathrm{MeV}$ for $\Delta m \simeq 1.25 \mathrm{GeV}$ ). A $4.5 \mathrm{MeV}$ smearing is applied to the three-body phase-space profile to account for the width of the reconstructed $\phi$ signal.

There are clearly enhancements with respect to the phase-space continuum at the kinematic threshold and around $\Delta m \approx 1.2 \mathrm{GeV}$; the fitted mass of the first structure is $m=(4148.2 \pm 2.0$ (stat. $) \pm$ 4.6(syst.) $) \mathrm{MeV}$, whereas that of the second structure is $m=(4316.7 \pm 3.0$ (stat. $) \pm 7.3$ (syst.) $) \mathrm{MeV}$. In the region of these two structures the three-body phase-space profile is certainly conservative since it lies above the background profile obtained by an event-mixing technique. The signal yields associated to the two structures are $355 \pm 46$ and $445 \pm 83$ respectively. On the same figure the result of a $2 \mathrm{D}$ simultaneous fit of both $B^{+}$invariant mass and $\Delta m$ distributions, with implicit background subtraction and efficiency correction, is shown as well. Several checks have been done to validate the robustness of the two structures. Not only each selection requirement has been varied but the whole analysis has been repeated with tighter selection criteria that lowered the combinatorial background level by a factor of ten while retaining $40 \%$ of the $B$ signal candidates.

While the first structure is consistent with the $Y$ (4140) by CDF [23][24], the additional peak is mass-shifted with respect the $Y(4274)$ by CDF [24]. A naive estimate of the yields' ratio (of $Y(4140)$ with respect to $B^{ \pm}$) is consistent with that of CDF [24] and the upper limit by $\mathrm{LHCb}$ [25]. The first structure is observed with a significance exceeding $5 \sigma$; this is evaluated either by performing a likelihood ratio test and applying Wilk's theorem, and by performing toy-MC simulation to calculate a $p$-value for the background fluctuations alone to give rise to a signal as 
significant as that seen in the data. The systematic uncertainties' evaluation has been obtained by changing signal and background fit models, $\Delta m$ binning, and varying the mass resolution, the efficiency correction and the selection criteria.

The background-subtracted $\phi K^{ \pm}$invariant mass distribution presented in Figure 3 (right), shows an excess with respect to the pure phase-space profile in the $1.7-1.8 \mathrm{GeV}$ region where large resonances, such as the interfering $K_{2}(1770)$ and $K_{2}(1820)$, may appear. The reflections studies subsequently carried out (see Figure 4) suggest that the first structure is not correlated to eventual $\phi K$ resonances; instead the second seems to be partially correlated.

Unfortunately the analysed data sample did not allow to extract a pure enough $B^{ \pm}$signal with sufficient statistics useful to perform an amplitude analysis needed to take into account the helicity configuration of the decay products. Very recently LHCb [27] provided the results of an amplitude analysis of this decay mode exploiting the full LHC Run-I data: the data cannot be described by a model that contains only excited kaon states decaying into $\phi K^{ \pm}$and four $J / \psi \phi$ structures are observed. Even if the one closest to the mass threshold is slightly better described as a $D_{s}^{ \pm} \bar{D}_{s}^{\mp}$ cusp, its resonant $\left(1^{++}\right)$interpretation is also possible with a mass consistent with the $Y(4140)$ but with a much larger width than the one estimated by CMS. This $J / \psi \phi$ structures' puzzle needs LHC Run-II data to be solved.

\section{CONCLUSIONS}

Two relevant contribution by the CMS Collaboration to the exotic quarkonium spectroscopy have been reviewed. The differential and total prompt production cross section of the $X(3872)$ have been measured in $p p$ collisions at $\sqrt{s}=7 \mathrm{TeV}$ and compared with available theoretical predictions based on an S-wave molecular model. The measurement of the $X(3872)$ nonprompt fraction confirmed that the total cross section is quite dominated by prompt production. The study of the dipion mass spectrum for $X(3872) \rightarrow J / \psi \pi^{+} \pi^{-}$confirms the presence of the $\rho^{0}$ as rather preferred intermediate state.
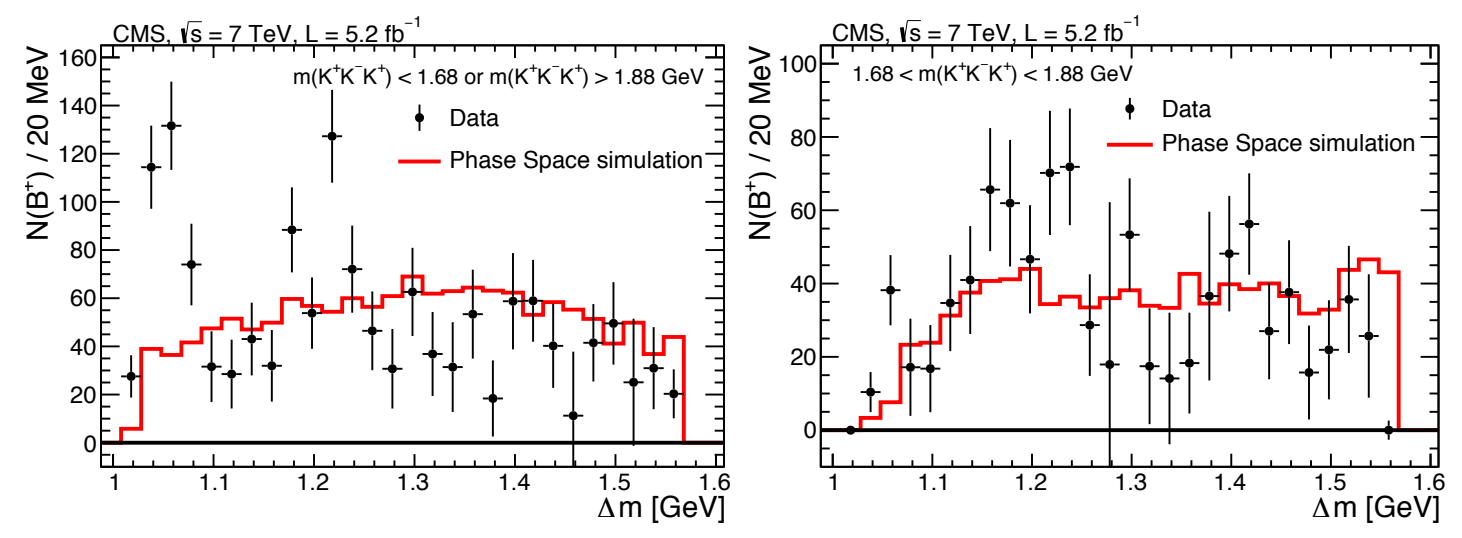

Figure 4: The number of $B^{ \pm} \rightarrow J / \psi \phi K^{ \pm}$candidates as a function of $\Delta m$ either (left plot) requiring the exclusion of the $m\left(\phi K^{ \pm}\right)$region from 1.68 to $1.88 \mathrm{GeV}$ showing an excess with respect to the phase-space profile and conversely (right plot) considering only this region. 
The observation of a peaking structure, close to the kinematical threshold, in the $J / \psi \phi$ mass spectrum for $B^{ \pm} \rightarrow J / \psi \phi K^{ \pm}$decays is consistent with a previous indication by CDF. Very recently LHCb confirmed it at a similar mass value but found a larger width. The data analysed by CMS did not provide a sufficiently large and pure sample for an amplitude analysis. A re-analysis extended to the full available data sample should allow this proper study.

\section{References}

[1] R. Jaffe, Phys. Rev. D 15 (1977) 1

[2] Belle Collaboration, Phys. Rev. Lett. 91 (2003) 262001

[3] N. Brambilla et al., Eur. Phys. J. C 71 (2011) 1534; E. Braaten et al., Phys. Rev. D 90 (2014) 014044; S. L. Olsen, Front. Phys. 10 (2015) 2, 121; A. Esposito et al., Int. J. Mod. Phys. A30 (2015) 1530002

[4] CMS Collaboration, JHEP 04 (2013) 154

[5] CMS Collaboration, Phys. Lett. B 734 (2014) 261

[6] CMS Collaboration, Phys. Lett. B 727 (2013) 57

[7] CMS Collaboration, CMS-PAS-BPH-16-002, https://cds.cern.ch/record/2204918

[8] D0 Collaboration, Phys. Rev. Lett. 117 (2016) 022003

[9] CDF Collaboration, Phys. Rev. Lett. 93 (2004) 072001

[10] LHCb Collaboration, Eur. Phys. J C72 (2012) 1972

[11] ATLAS Collab., https://cds.cern.ch/record/2160169/; see P. Iengo's contribution in these proceedings.

[12] CMS Collaboration, JINST 3 (2008) S08004

[13] LHCb Collaboration, Phys. Rev. Lett. 110 (2013) 222001

[14] LHCb Collaboration, Phys. Rev. D92 (2015) 011102

[15] CMS Collaboration, JHEP 02 (2012) 011

[16] Particle Data Group, Review of particle physics (RPP), Phys. Rev. D 86 (2012) 010001

[17] P. Artoisenet and E. Braaten, Phys. Rev. D 81 (2010) 114018

[18] Bignamini et al., Phys. Rev. Lett. 103 (2009) 162001

[19] CDF Collaboration, Phys. Rev. Lett. 96 (2006) 102002

[20] Belle Collaboration, Phys. Rev. D 84 (2011) 052004

[21] BaBar Collaboration, Phys. Rev. D 74 (2006) 071101, Phys. Rev. Lett. 102 (2009) 132001

[22] Babar Collaboration, Phys. Rev. Lett. 101 (2008) 082001

[23] CDF Collaboration, Phys. Rev. Lett. 102 (2009) 242002

[24] CDF Collaboration, arXiv:1101.6058 (2011)

[25] LHCb Collaboration, Phys. Rev. B 85 (2012) 091103

[26] M. Pivk and F. LeDiberder, Nucl. Instrum. Meth. A 555 (2005) 356

[27] LHCb Collaboration, arXiv:1606.07898 (2016); see R. Cardinale's contribution in these proceedings. 